\begin{tabular}{cc}
\hline International Journal of Engineering \&Technology, $7(1.7)(2018) 34-37$ \\
SPC & Website: ww.sciencepubco.com/index.php/IJET \\
Research paper & International Journal of Engineering \& Technology \\
\hline
\end{tabular}

\title{
Survey of rice seed quality analysis for varietal purity estimation by using image processing techniques
}

\author{
S. Durai ${ }^{1 *}$, C. Mahesh ${ }^{2}$, T. Sujithra ${ }^{3}$, A.Suresh ${ }^{4}$ \\ ${ }^{1}$ Research Scholar, Department of Computer Science and Engineering, School of Computing, Vel Tech Rangarajan \\ Dr. Sagunthala R\&D Institute of Science and Technology, Avadi, Chennai-62, TamilNadu, India \\ ${ }^{2}$ Associate professor, Department of Information Technology Vel Tech Rangarajan Dr. Sagunthala R\&D \\ Institute of Science and Technology, Avadi, Chennai-62, TamilNadu, India \\ ${ }^{3}$ Assistant Professor, Department of Computer Science and Engineering, School of Computing, Vel Tech Rangarajan \\ Dr. Sagunthala R\&D Institute of Science and Technology, Avadi, Chennai-62, TamilNadu, India \\ ${ }^{4}$ Professor \& Head, Department of Computer Science and Engineering, Nehru Institute of Engineering and Technology, \\ T.M.Palayam, Coimbatore-641105, TamilNadu, India \\ *Corresponding author E-mail: duraitrichy@gmail.com
}

\begin{abstract}
In south India rice is the major food source and in agriculture, rice production covers more than 70 percentages of entire forming. But in recent the production only from south India not enough to satisfy the need of all, such a huge demand is there. The better production comes from the selection of good seeds. Up to now formers depend on two factors for selecting better seeds, One is the brand which is approved by some quality standards and second one is analyzed manually by experienced people. Both are risky one, we are not pretty much sure the accuracy of analyze. The second one is seeing and feeling. The inspection is not consistent also very time consuming. In the other way we can use computer vision technology to analyze the quality of the seeds. In recent years many of the big industries they are using computer vision technology with Digital Image Processing for many of the applications. In this Paper we are going to discuss the different seed quality analyzing methods and accuracy of result also. Moreover there are different factors and features are there for it, here we are going to study about varietal purity estimation by different methods.
\end{abstract}

Keywords: Computer vision; rice seed analysis; Quality factors of rice seed; rice seed varietal purity.

\section{Introduction}

Assuring the rice seed quality is the biggest and difficult test for the massive rice exporting countries. India, Thailand, US and Vietnam are the topmost countries which exporting rice. Rice seeds purity decides the yield of the grains, inferiority seeds not only affects yield its reduce the market rate of the product. So the quality of the rice is the basic test for all the rice makers which lies in selecting the good seeds. When we are doing it manually it takes lots of time and there is no guarantee for the accuracy because manual testing is like what they feel and see. I $\mathrm{n}$ other way when considering the physical characteristics of rice seed like shape, size, color, length and width etc, we can use computer systems to analyze the quality of the seed.

Figure 1 refers the life cycle of the rice seed, the fundamental and the basic important is seed selection and its quality [8]. Seed is the establishment of any rice edit. It must be developed, collected, and prepared accurately for best yield and quality outcomes.

\subsection{Factors to classify seed}

- $\quad$ Varietal purity

- $\quad$ Seed Viability

- Moisture content

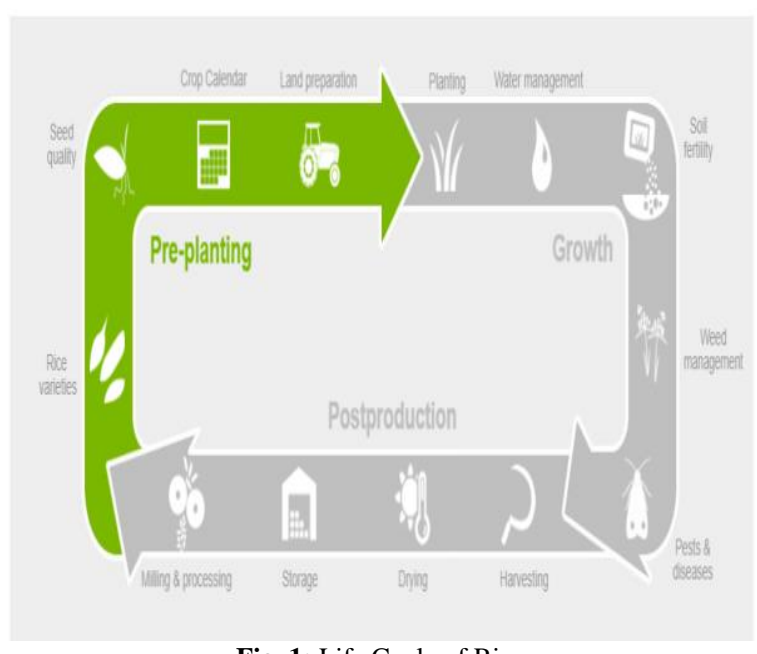

Fig. 1: Life Cycle of Rice.

1.1.1. Varietal purity depends upon the following conditions

- Other mixed varieties

- Red rice seeds

- Germination capacity of the seed

Germination $\%=($ No. of Seeds germinated $) /$ (No. of seeds taken for the sample) 
- Weed seeds and other crop seeds

Weed $\%=$ (weight of weed) / (Weight of seeds taken for the sample)

- Unwanted materials like stones, dust, soil etc.

Unwanted materials $\%=$ (Weight of Unwanted materials) $/($ Weight of seeds taken for the sample)

- Moisture content

\subsubsection{Seed viability calculated by the following factors}

The suitability of seed in the field is dictated by its germination potential, force, and moisture level. Then to get the good seed is in the form of, Purchase confirmed seed that is unadulterated and named, Get rancher created great seed, select your own great seed.

\subsubsection{Moisture content is measured by}

The amount of water contained in the rice seed fix the moisture content of the rice seed. For good seed related to moisture content must be less than 14 percentages. By using resistance type moisture meter we can measure moisture content at the time of harvesting seeds, it gives results quickly but we can use it only for small samples. For large set of samples we need capacitive moisture meters, they are more expensive.

\subsection{Rice seed quality assessment kit}

For estimating all the above factors we discussed IIRI developed assessment Kit. It consists the following, but it's too costly and each component test only a particular test.

- $\quad$ Graduated Cylinder (Flask)

- Scale

- Caliper

- Infrared Thermometer

- IRRI Moisture Tester

- Indented Sheet Grader

- Magnifier

- Rice Milling Chart

Figure 2.0 shows the Rice seed quality assessment kit developed by IIRI rice knowledge bank.

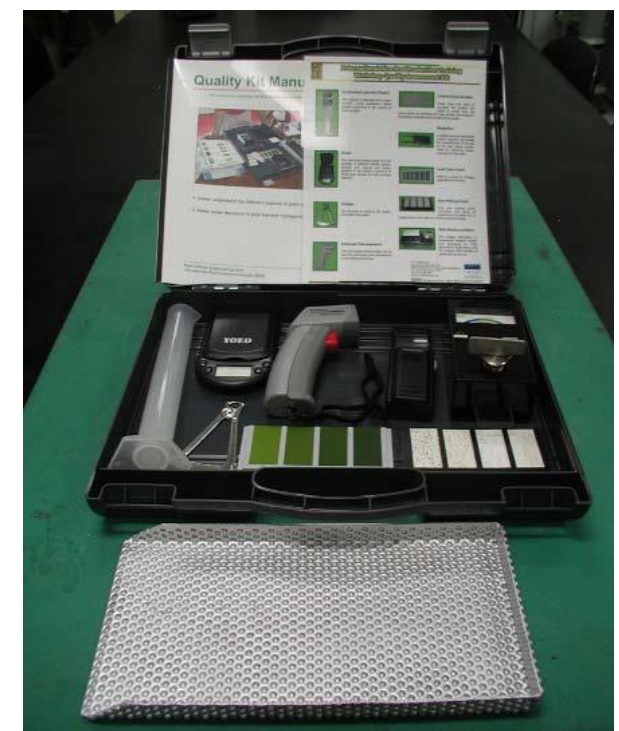

Fig. 2: IIRI Rice Seed Quality Assessment Kit.

\section{Basic hardware setup requirements}

The hardware configuration of computer based machine vision system is common for all systems Figure 3.0. It consists of the following

- An illumination device, which illuminate the samples under test.

- To acquire image a solid state CCD camera.
- A frame-Grabber

- A personnel computer

- A high resolution color monitor

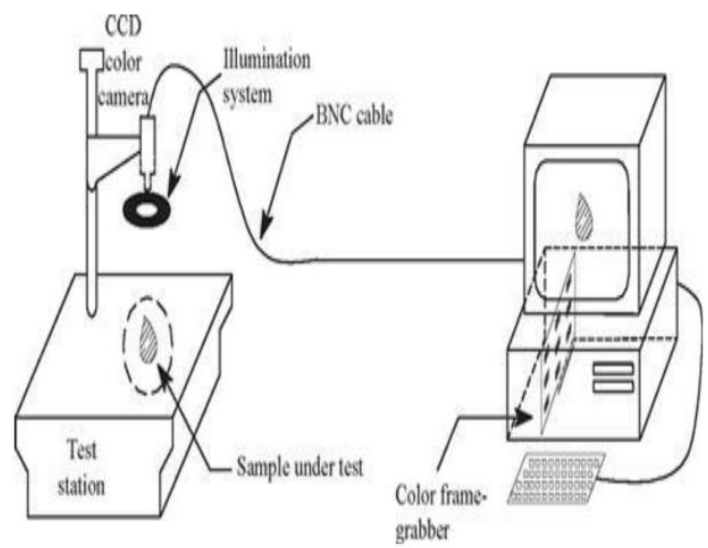

Fig. 3: Standard Hardware Setup to Get Sample Data.

\section{Experimental results}

\subsection{Samples of rice seed varieties}

[1] They took six rice seed varieties ey7954, syz3, xs11, xy5968, xy9308, z903 for analysis and morphological features were extracted. They used seven colors and fourteen morphological properties of rice seed. [2] They proposed an automatic identification and classification of rice seeds of different varieties using machine vision system. They took five different varieties of rice seeds Xiannong, Jinyougui, You166, Xiannong, Medium you. They computed color Spaces and RGB (Red,Green, Blue) color features. To identify the variety of rice seed they used backforward neural network.

[5]They considered Six normal developed rice assortments in Northern Viet Nam, viz. BC-15, Hương thơm 1, Nếp-87, Q-5, Thiên ưu-8, Xi-23. Grain seeds were tested from a rice seed manufacture organization where the rice was produced also, gathered after specific conditions for standard rice seeds generation.[6] An assessment of the characterization exactness of surface and shape highlights and neural system was done to analyze and classify four rice grains, to be specific, Karjat-6(K6), Ratnagiri-2(R2), Ratnagiri-4(R4), and Ratnagiri-24(R24).

[7]Khunkhett and Remsungnen proposed identification of pure rice breeds by considering destruction factor of rice seed. They took Khao Dawk Mali 105 rice seed for analyze it. They followed four seed levels for each variety of rice,

- Foundation seed

- Commercial Seed

- Breeder Seed

For the quality of rice they considered six standard requirements,

- Good seed

- Contamination

- Germination

- Moisture content

- Other species

- Red rice seed

\subsection{Image acqusition}

[1] They used charge coupled cadget (CCD) camera with TMC7DSP display of $640 \times 480$ pixels for capturing the images. The view of the field was $12 \times 9 \mathrm{~mm}$. The spatial measurement was $0.19 \mathrm{~mm} /$ Pixel. To decrease the impact between the seed and light they used a dark light chamber between the examples table and the focal point. All seeds taken for the test were accepted as a good seed tested by human. They used white as the background. [2] Figure 4.0 shows the proposed method of A G. OuYang. The 
rice seeds were dropped out from the container and distributed over foreordained lattice which then move over the belt for taking images. The moving belt which contains seed is placed under the camera and is captured by CCD cameras. The cameras are already attached with PC for storing images. And the taken images were sent to picture preprocessing unit in PC. They used the background as dark and the surface color was dim green.

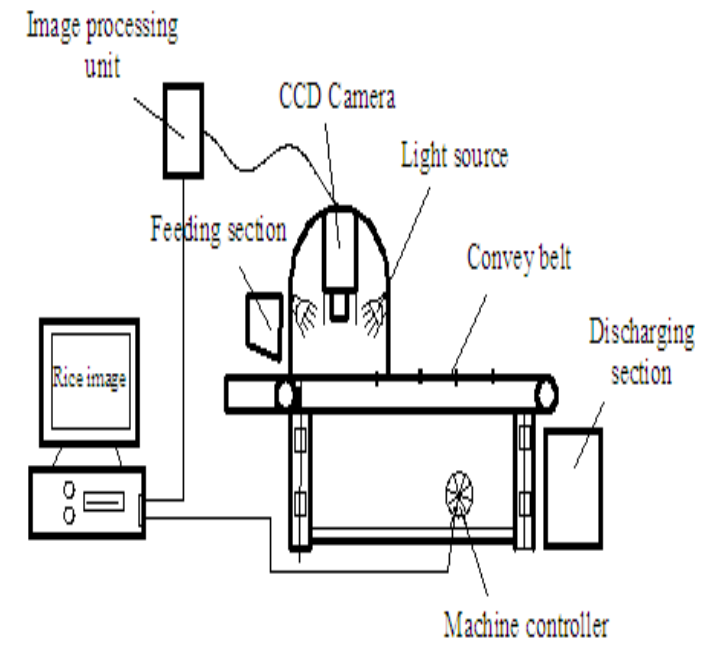

Fig. 4: Automatic Rice Seed Variety System.

[5] They used CMOS image (NIKON D300S) camera with determination of $640 \times 480$ pixels was used to get the images. They set up samples with a white table as background for capturing photos. Rice seeds are distributed inside the area of $10 \times 16 \mathrm{~cm}$.. Each picture taken by this imaging framework contains around 30 to 60 seeds. We at that point isolated rice seed pictures and understood the picture division.[6] They used Sony make 18.9 megapixels computerized camera, dark material sheet for background, and photos of the paddy seeds. The Seeds were collected from the seed Testing Laboratory Pune, India, for the grain tests. The unclean business tests of four paddy grains, K6, R2, R4, and R24, were gathered.

[7] They follow the following hardware materials for the getting the input. They used canon MP287 with 1200 X2400 dpi scanner and Compaq Presario V3000 Dual - core processor notebook computer system.

\subsection{Image segmentation}

Image segmentation is the important and the basic step in the digital image processing. We are going to subdivide the image into set of parts until we are getting the clear or segmented for testing. [1] The edge esteem is created agreeing to the consequences of the histogram examination and was steady for a similar situation conditions. In this examination, it was discovered that the blue esteem was exceptionally distinctive between the foundation and the articles. A settled limit esteem decided from the histogram of the blue plane could isolate the rice seed from its background. Figure 5.0 a shows the segmented sample. [2] The consequence of division is typically a twofold image. A twofold image contains just two kinds of pixels: the pixels having a dark level estimation of either 0 or 1 . Objects of intrigue are disengaged in pixel accumulations at a dark level of 1 , which are as yet crude information requiring a reasonable depiction for further PC handling. Figure $5.0 \mathrm{~b}$ shows the segmented sample.

[6] The image examination programming was created in Matlab adaptation 7.12.0.635 (R2011a). With a specific end goal to remove question highlights, image division and any essential morphological separating were finished. . Figure $5.0 \mathrm{c}$ shows the segmented sample. [7] They followed sequence of three steps for processing it. They are getting the sample by using scanner, they used some pre-processing methods to enhance it, Binarization of the gray scale image. Figure $5.0 \mathrm{~d}$ shows the segmented sample.

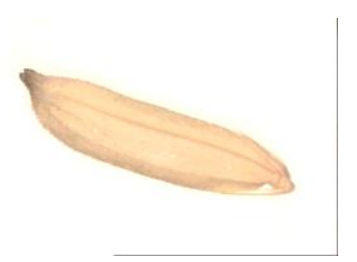

Fig. 4: A) Sample Segmented Image Xy5968.

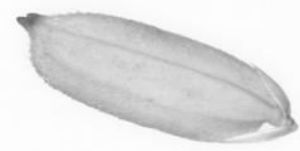

Fig. 4: B) Sample Segmented Image of Xiannong.
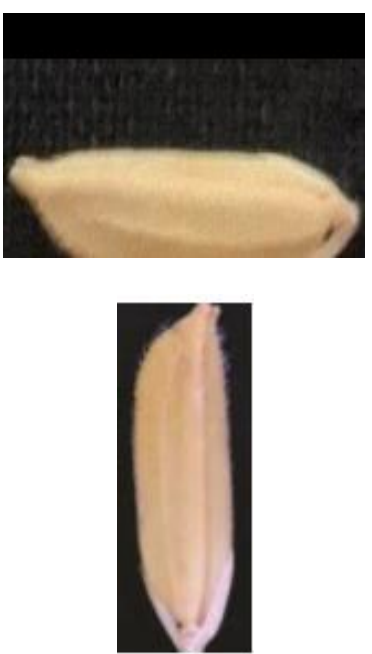

Fig. 4: C) Sample Segmented Image of R-2.

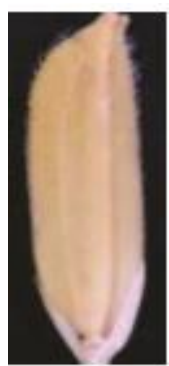

Fig. 4: D) Sample Segmented Image of Khao Dawk Mali 105.

\subsection{Feature extraction}

[1] They used color feature and morphological feature for analyzing rice seed. Using Matlab they develop algorithm to extract R, G, B color bands of an image and Hue $(\mathrm{H})$, Saturation (S) and Intensity of an image were calculated. In morphological feature they extracted Areal, length, Width, major axis length, minor axis length, Thinness ratio and Aspect ratio. [2] The entire segment was divided, and the developing life territory what's more, the fine territory were approximated. Each fragmented question was examined to get its zone. The pixel zone was processed by calling the Matrox on-board factual routine to check the number of pixels for each question. The main component $(\mathrm{x})$ was the proportion of the sound zone to the region of the entire area. The second element (y) was the proportion of the sound region to the territory of the approximated incipient organism part. The second element was the more vital if the guess of the fetus part was precise. The two highlights were exhaustively utilized for the arrangement.

[5] In this work, they researched four component types that could be considered as delegate of a fundamental gatherings of highlights: worldwide highlights (morphological highlights, shading, surface, GIST). Morphological highlights are the most common highlights to portray the state of the protest in image. Shading and surface are extremely helpful to recognize objects when their 
shapes remain comparable. Substance is a worldwide element figured construct Gabor channel bank connected in light of the entirety image (Oliva and Torralba, 2001).

[6] They extracted texture feature for analyze the rice seed quality. Texture comprises of texture natives, texels, which are an adjoining set of pixels with some tonal as well as provincial property Texture can be described by power (shading) properties of texels and structure spatial connections of texels. Textures are very scale subordinate. Texture highlights are ordered as measurable/auxiliary what's more, linguistic. In factual texture examination, texture highlights are processed from the factual circulation of watched blends of powers at determined positions with respect to each other in the image. As indicated by the quantity of power focuses (pixels) in every blend, measurements are arranged into first-arrange, second-arrange, and higher-arrange measurements.

[7] They extracted physical data of rice seed like area, Perimeter, diameter and Histogram average, Red, Blue and Green are measured.

\subsection{Classification}

After feature extraction the next step is to classify the seeds by using any of the classification method. Here we referred some of the classification method that widely used for classify the segmented objects.

1) K- Nearest neighbor

$\mathrm{K}$-nearest neighbor $(\mathrm{KNN})$ is a strategy for arranging in light of $\mathrm{k}$ nearest neighbors and afterward predicts the class of a new specimen as the most incessant one happening in the neighbors. This strategy has been utilized generally in characterization issues since it is straightforward, compelling and non-parametric.

\section{2) Support Vector Machine}

The fundamental thought of help vector machine (SVM) is to locate an ideal hyper-plane for straightly distinct examples in a high dimensional space where highlights are mapped onto. There is more than one hyper-plane fulfilling this model. The undertaking is to recognize the one that amplifies the edge around the isolating hyper-plane. This finding depends on the bolster vectors which are the information indicates that lie nearest the choice surface and have coordinate bearing on the ideal area of the choice surface. SVMs are stretched out to order designs that are not directly divisible by changes of unique information into new space utilizing piece work into a higher dimensional space where classes turn out to be straightly divisible. SVM is a standout amongst the most effective and broadly utilized as a part of classifiers.

3) Random Forest

Breiman proposed RF- Random forest classification method by constructing sequence of decision trees. For every decision tree random forest uses variety of different bootstrap samples and changes how the trees are constructed and how classification was done. Every node divided by using the best subset and randomly chosen as the node and rapidly grows the tree to the highest extension without removing any node. RF aggregates all outputs to predicting the new data. Compared to many other classification techniques, RF is efficient, quickest with a huge amount of data and performed well. They conclude that RF is best among discriminate analysis, neural networks, support vector machines and is portable over-fitting.

\subsection{Result discussion}

[1] They implemented model with six rice seed varieties and they got the accuracy $90.00 \%, 88.00 \%, 95.00 \%, 82.00 \%, 74.00 \%$, $80.00 \%$ for ey 7954, syz3, xs 11, xy5968, xy9308, z903 respectively.[2] The rectify classification rates for five rice assortments were: No.5 'Xiannong' of $99.99 \%$, 'Jinyougui' of $99.93 \%$,'You166' of $98.89 \%$, No. 3 'Xiannong' of $82.82 \%$ and 'Medium you' 463 of $86.65 \%$, individually. In view of the outcomes, it was inferred that the framework was sufficient to use for examination of assortments of distinctive rice seeds in view of their appearance characters of seeds.[5] Their trials have illustrated that the normal preci- sion of our classification framework can achieve $90.54 \%$ by utilizing Random Forest strategy with a essential element extraction system. This outcome can be utilized for building up a PC helped machine vision framework for computerized evaluation of varietal immaculateness of rice seeds. [7] They showed over 98 percentage of accuracy of test by using digital image processing technique and the whole system achieved over 83 percentages.

\section{Conclusion}

In this paper we studied the alternate method for analyzing the seed quality in terms of using machine-vision system by using Digital image processing methods and classification. And we discussed about the hardware setup and requirements of each proposed methods. Detail study about how to collect the data set, image segmentation and different Feature extraction methods for each proposed methodology. We discussed about the different classification methods widely used for classifying the data sets with the help of extracted features. Finally we conclude the accuracy percentage they obtained for each proposed methodology for the corresponding rice seed variety. In feature we strongly recommend that instead of CCD camera images we can implement the same methods by using MRI images or IR images for better accuracy and better performance.

\section{References}

[1] Z.-y. Liu, F. Cheng, Y.-b. Ymg, and X.-q. Rao, "Identification of rice seed varieties using neural network," Journal of Zhejiang University Science. B, vol. 6, no. 11, pp. 1095-1100, 112005.

[2] A G. OuYang and R. j. Gao et al, "An automatic method for identifying different variety of rice seeds using machine vision technology;' in 2010 Sixth Int. Con! on Natural Computation, vol. 1, Aug 2010, pp. 84--88.

[3] P. T. T. Hong and T. T. T. H. et al., "Comparative study on vision based rice seed varieties identification," in Knowledge and Systems Engineering (KSE), 2015 Seventh Int. Con! on, Oct 2015, pp. $377-$ 382

[4] D.-W. Sun, Computer Vision Technology for Food Quality Evaluation. Elsevier, 2008.

[5] Phan Thi Thu Hong, Tran Thi Thanh Hai, Le Thi Lan, Vo Ta Hoang and Nguyen Thi Thuy "Identification Of Seeds Of Different Rice Varieties Using Image Processing And Computer Vision Techniques" J. Sci. \& Devel. 2015, Vol. 13, No. 6: 1036-1042

[6] Archana Chaugule and Suresh N. Mali "Evaluation of Texture and Shape Features for Classification of Four Paddy Varieties" Hindawi Publishing Corporation Journal of Engineering Volume 2014, Article ID 617263, 8 pages https://doi.org/10.1155/2014/617263.

[7] S. Khunkhett, T. Remsungnen "Non-Destructive Identification Of Pure Breeding Rice Seed Using Digital Image Analysis" JICTEE2014

[8] S.J. Mousavirad, F. A. Tab, and K. Mollazade, "Design of an Expert System for Rice Kernel Identification using Optimal Morphological Features and Back Propagation Neural Network," International Journal of Applied information systems, vol. 3, pp. 33-37, 2012.

[9] S.Durai, M.Thanjai Vadivel, T.Sujithra "Grading of Rice Quality by Chalky area analysis Using Simple Digital Image Processing Techniques" International Journal of Pure and Applied Mathematics Volume 114 No. 12 2017, 657-665.

[10] http://www.knowledgebank.irri.org/ 